\title{
A Compiler for Natural Semantics
}

\author{
Mikael Pettersson \\ Department of Computer and Information Science \\ Linköping University, S-581 83 Linköping, Sweden \\ E-mail: mpelida.liu.se
}

\begin{abstract}
Natural semantics is a formalism used for specifying both semantics and implementations of programming languages. Until recently, no practical implementation of the formalism existed. We have defined the Relational Meta-Langurge, RML, as an executable specification language for natural semantics. After a brief outline of the language, we describe the compilation strategy used by our rml2c compiler: transformations are applied to minimize non-determinism, and a continuationpassing style form is produced and simplified. Finally the CPS is emitted as low-level $\mathrm{C}$ code, using an efficient technique for implementing tailcalls. We also present performance measurements that support our choice of compilation strategy.
\end{abstract}

Keywords: natural semantics, determinacy, continuations, tailcalls.

\section{Introduction}

Natural Semantics is a successor to Structural Operational Semantics that has become a popular tool for specifying type systems, dynamic (interpretive) semantics, and compilation $[9,18,20,25]$. Until recently, the only implementation of the formalism was the Typol language in the Centaur programming environment $[10,17]$. However, that implementation is both very inefficient, and does not support stand-alone executables.

We have a defined a meta-language for Natural Semantics, RML, with the intention of producing a practical compiler for it. Efficient code was the foremost goal, followed by the ability to produce code that could be combined with other code, hand-written or generated, to form stand-alone applications.

In this paper we describe the main aspects of the rml2c compiler, which compiles RML to efficient $\mathrm{C}$ code. Our contributions are:

- We outline the features of Natural Semantics and the RML specification language.

- We describe a reasonably straightforward compilation strategy for RML that has proven to be very effective for several non-trivial specifications.

- Compiling tailcalls to $\mathrm{C}$ code is a problem faced by many compilers for highlevel languages; we describe a new strategy that is both correct and efficient.

Section 2 starts by giving a brief overview of natural semantics and RML. Section 3 then discusses the compilation strategy, and some alternatives, followed in section 4 by a description of the high-level transformations employed in the 
first compilation phase. Then follows section 5 with a brief discussion of the continuation-passing style intermediate representation used. The back-end emits low-level $\mathrm{C}$ code, but in doing so needs to emulate tailcalls. Section 6 starts with a brief overview of known techniques, before detailing a new solution we have developed. Then follows a summary of our benchmark results in section 7 . Section 8 compares this with related work, and section 9 concludes.

\section{Overview of Natural Semantics and RML}

Natural semantics is a formalism frequently used to specify programming languages in general, type systems, special properties, and transformations between representations $[9,18,20]$. Specifications consist of three parts: declarations of the types of objects involved (typically in the form of abstract syntax), declarations of the judgements (logical formulas) involved, and axioms and inference rules defining those judgements. The end result is a Gentzen-style natural deduction system (a sequent calculus [12]) for an application-specific logic.

The Relational Meta-Language, RML, is a straightforward realization of the description above. Data type declarations are identical to those found in Standard ML, and 'relations' (our terminology for groups of related inference rules) are written textually almost as they are on paper. An ML-like polymorphic type system is present for static type checking. The operational interpretation of specifications is roughly as in Prolog. To prove a judgement, a top-down search through the program is performed to find a relevant inference rule. Then a leftright execution of the premises of that rule is done, after which the judgement is considered 'proved'. As in Prolog, we refer to relations as procedures when taking an operational view.

In contrast to Prolog, the search rule is defined to be determinate, which means that once a proof is found for a judgement, no attempts will be made to find alternative proofs. The full power of backtracking is still present during proof construction.

A small example specification is listed in figure 1. It contains type declarations for expressions and environments, and a relation eval (env, exp) $\Rightarrow$ int describing the relationship between environments, expressions, and values. Pragmatically, we use the relation to evaluate expressions in order to retrieve their values.

\section{Implementation Strategy}

The compilation process is a multi-stage pipeline, with appropriate optimizations applied at each level:

1. Source code is translated to a form reminiscent of first-order logic, FOL. A left-factoring transformation is applied to reduce the usually enormous amount of non-determinism present in specifications. 


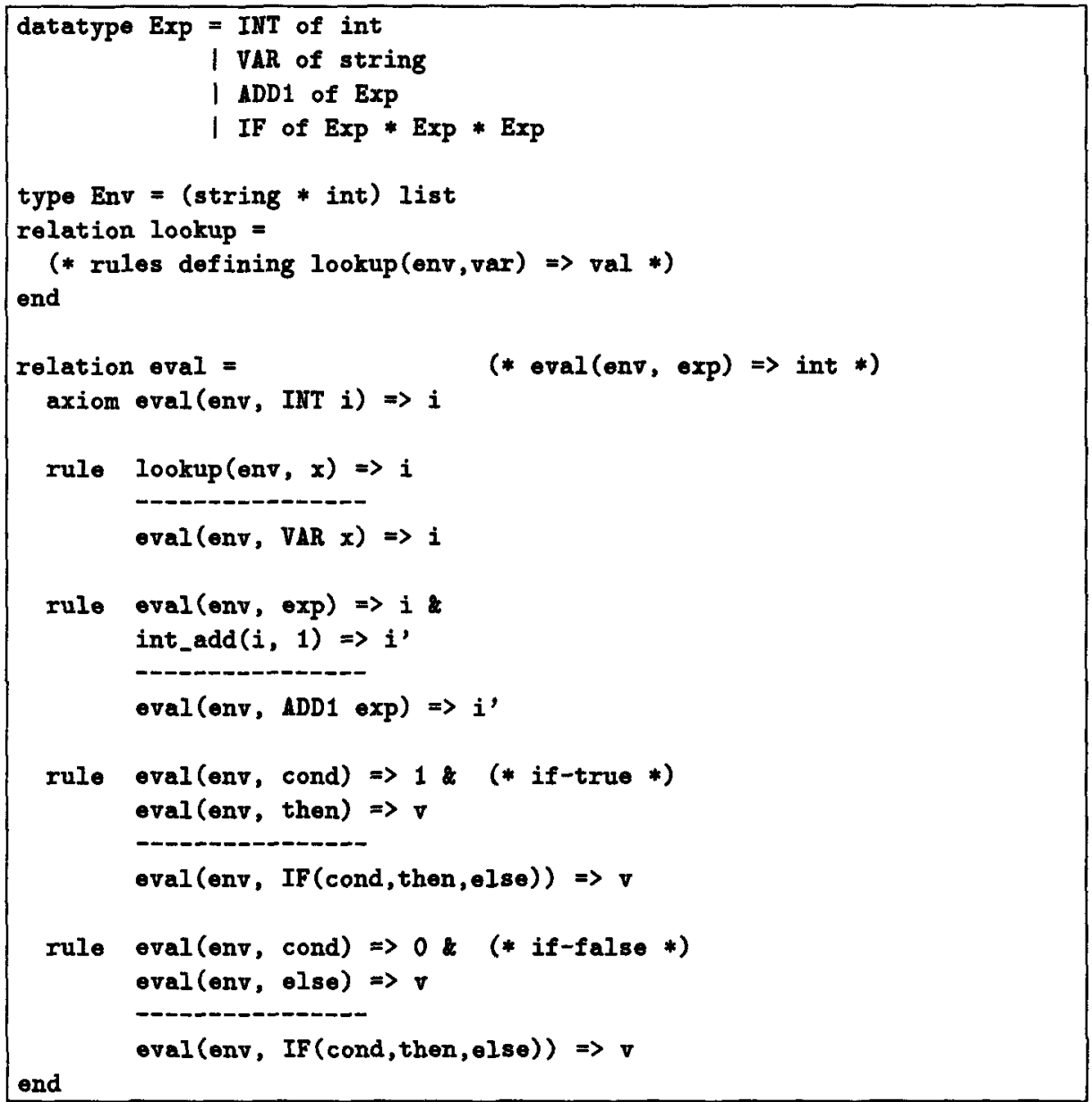

Fig. 1. Small RML example

2. Then the FOL form is translated to a continuation-passing style functional form, CPS. Inlining of procedures and continuations is performed, as is constant propagation and other simple optimizations.

3. A low-level Code representation is produced in which continuations are replaced by concrete data structures, and data structure creation is made explicit. A copy-propagation optimization is applied to remove some redundancies introduced by the code to pass parameters when tailcalls are emulated.

4. The final step prints the low-level form as $\mathrm{C}$ code, compiles it, and links with a small $(\approx 2600$ lines $)$ runtime system.

The compiler is written in 11569 lines of Standard ML.

Absent from this description is any mention of Warren's Abstract Machine (WAM) [2], which is a standard starting point for logic programming language 
implementations. There are two reasons for this. First, its instructions are very low-level, which means that high-level optimizations would be difficult to express for it, and it is not certain that they would be effective. Second, the instructions are complex, hiding many smaller operations inside. This in turn prevents useful low-level optimizations.

In an earlier paper [22], we showed that a continuation-passing style denotational semantics for RML has operational properties that correspond closely to the WAM. In essence, we make use of a WAM-like model of the control and trail stacks, but ignore its instruction set.

\section{Improving Determinacy}

Many natural semantics specifications describe deterministic processes, e.g. type checking, evaluation, or translation. However, neither the formalism nor RML has local control-flow operators such as conditionals. Choices have to be expressed as separate inference rules.

Consider for instance the expression evaluator in figure 1, in particular the two rules defining IF-expressions. Interpreted naively, the first rule calls for the pattern-matching of the arguments, followed by evaluation of the predicate. If the predicate is not true (1), the first rule fails, backtracking takes us to the next rule, and the pattern-matching and evaluation is done all over. Obviously, this can be very inefficient.

While rules like these can be rewritten to be more efficient, this is not something we want specification writers to have to deal with. Instead, the compiler performs a left-factoring transformation that converts these rules into the obvious conditional.

\subsection{Left-factoring}

We call this transformation left-factoring because execution of relations is much like top-down parsing, and the transformation is similar to left-factoring of context-free grammars.

After type checking, relations are translated into a form that is a disjunctivenormal form of a kind of first-order logic; we call it FOL. First, every inference rule becomes a conjunction of its explicit and implicit goals. For instance, the first rule for the conditional above would become (we use Lisp-like notation for these forms):

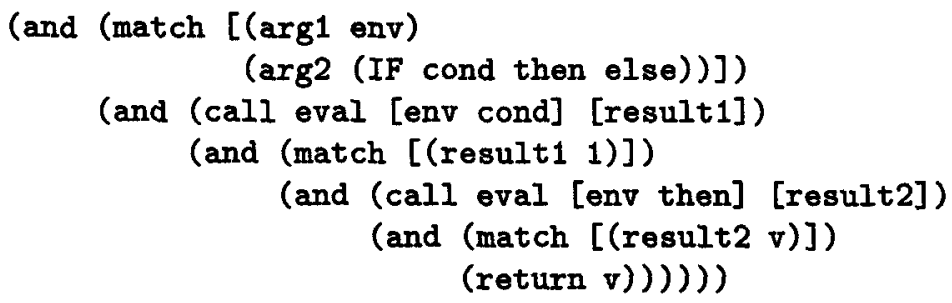


I.e., the arguments are pattern-matched, a call is performed, the result is patternmatched, a new call is done and its result is pattern-matched, and finally a single value is returned. The second rule would be similar. Then the disjunction of all these conjunctions is formed.

Left-factoring attempts to perform the following transformations:

- Consider a disjunction of the form:

$$
(A \wedge B) \vee(A \wedge C)
$$

If the left $A$ succeeds, but either $B$ fails, or failure occurs later, then the right $A$ will be executed during backtracking. This second execution is clearly redundant ${ }^{1}$. On the other hand, should the left $A$ fail, then there is no point in trying to execute the right $A$. Therefore, this form is rewritten to:

$$
A \wedge(B \vee C)
$$

This is reminiscent of both one of the basic laws of logic and the familiar left-factoring of context-free grammars.

- In a disjunction of the form:

$$
(A \wedge B) \vee(\neg A \wedge C)
$$

the two disjuncts are mutually exclusive: if the left one succeeds, the right one cannot possibly succeed. This form is rewritten to use a logical if-then-else operator:

if $A$ then $B$ else $C$

- Match operators are introduced to make implicit pattern-matching operations explicit ${ }^{2}$. If both parts of a disjunction $(M \wedge A) \vee\left(M^{\prime} \wedge B\right)$ start by using the match operator on the same variables, then the two matches are combined into a larger case construct. The branches of the case are the remainders of the disjuncts, i.e. $A$ and $B$.

For example, after left-factoring, the two rules for the conditional would have been rewritten to:

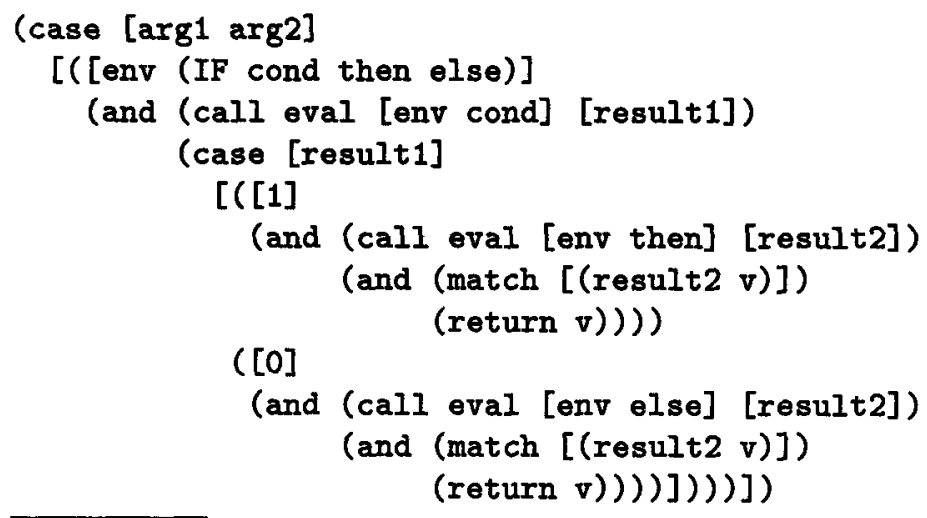

\footnotetext{
${ }^{1}$ In a declarative language, that is.

${ }^{2}$ But they are not 'compiled' to simple code; that is done in the translation to CPS.
} 
This form no longer contains any explicit disjunctions. Later on, the patternmatch compiler will discover that there is no overlap between the cases in the inner case, so the implicit disjunction there is also eliminated. The result is completely deterministic code.

\subsection{Implementation}

Our compiler uses a term-rewriting system with 17 rules to specify these and related transformations. Of the rules, 11 perform useful work, while the remaining ones make sure the terms are in a right-linear form recognizable by the useful rules.

The code, which is only 105 lines of Standard ML, also includes support for a limited form of higher-order unification. Some rules need to compare terms for equality, but equality must ignore the actual names of bound variables. Hence renamings must be performed on-the-fly.

\subsection{Limitations}

As indicated by the performance evaluation presented later, the left-factoring phase is very effective in removing unnecessary non-determinism. However, the purely syntactic nature of the transformations causes some cases to be missed.

Some primitive language operators are known to be mutually exclusive. For instance, two rules, one containing $x<y$ and one containing $x \geq y$, must be mutually exclusive. The current implementation knows nothing of primitive operators, and so will not be able to introduce a conditional in this case. This problem, while not frequent, does occur occasionally.

Also, even if two user-defined relations happen to be mutually exclusive, the compiler will not notice this since it never inspects the definitions of called relations. Luckily, typical specifications do not seem to suffer from this.

\section{Intermediate Code}

After the left-factoring optimizations, the FOL representation is translated into a continuation-passing style (CPS) representation. At the same time, a patternmatch compiler [21] is used to expand pattern-matching constructs into appropriate combinations of data structure inspections and tests.

Since RML has backtracking, the CPS transformation introduces two kinds of continuations, success and failure continuations. The former are used for normal calls and returns, while the latter are introduced at disjunctions as handles for backtracking [22]. Apart from this, the CPS representation is fairly conventional.

Our reasons for using CPS are several:

1. We obviously need to model and optimize both ordinary calls and returns, and backtracking. Continuations provide us with an easy way to do so. 
2. CPS has nice pragmatic features for compiler writers. It has a simple structure, and, being a $\lambda$-calculus, makes some optimizations (inlinings, constant and copy propagations) easy. Other lower-level optimizations can be left for the back-end, which in our case is a $\mathrm{C}$ compiler.

3. Although CPS is high-level, it has a simple interpretation in terms of machine resources. This makes the final translation to low-level code straightforward.

Our compiler only applies simple optimizations to its CPS representation, mainly constant propagation, copy propagation, and inlining of those continuations and procedures that have single unique invocation points. No static analysis is performed, except a simple per-module reference count analysis.

We refer the reader to the literature for further details concerning the practical issues involved $[3,19,22,27,28]$.

\section{Tailcalls in $\mathbf{C}$}

Implementations of high-level languages must often deal with tailcalls. A tailcall is a function call that occurs as the last action (the 'tail') in a function body. An implementation is properly tail-recursive if tailcalls do not cause net growth in stack usage. In effect, tailcalls are gotos that pass arguments.

Functional and logic languages typically have no primitive iteration constructs: instead recursive procedures and tailcalls are used to synthesize a variety of iteration schemes.

Some compilers, including rml2c, use a continuation-passing style representation of programs. In this representation, all non-local control transfers are tailcalls.

In both of these cases, it is important to implement tailcalls both efficiently and correctly, i.e. properly tail-recursively.

For portability reasons, many compilers choose to compile to low-level $\mathrm{C}$ code instead of machine code. This causes problems, since C's function calls are not guaranteed to be implemented tail-recursively ${ }^{3}$.

\subsection{Earlier solutions}

A number of techniques for implementing tailcalls have been proposed over the years, especially in the functional and logic programming communities; we summarize them below. We do not concern ourselves with approaches that do not guarantee proper tail-recursion.

In this section the term module is often used. By this we mean an individual compilation unit, usually just a single file with a set of declarations. In RML, a module is a language construct which simultaneously defines a number of types and relations, and declares the externally visible interface.

${ }^{3}$ Some $\mathrm{C}$ compilers recognize trivial cases, but the great majority never do anything special about tailcalls. 
In the plain dispatching technique, every label becomes a single function. When a label terminates with a tailcall, it returns the label to which control is to be transferred. A dispatcher is given an initial label. It calls the label, receives as the return value the next label to call, and loops ad infinitum. While this preserves tail-recursion, parameter passing cannot be done in a natural way. Instead, global variables are used to communicate arguments from caller to callee. This technique has been used many times $[24,27,28]$.

To eliminate the high overhead for parameter passing and control flow in the previous scheme, some compilers collect all code into a single $\mathrm{C}$ function [13]. This allows parameters to be located in local $\mathrm{C}$ function variables, perhaps even registers, and known control transfers become simple gotos. For unknown control transfers, a big switch statement is used ${ }^{4}$. This scheme is not really practical though, since it makes separate compilation impossible. Also, gathering all code into a single function makes that function extremely large, which in turn negatively affects compilation times and the $\mathrm{C}$ compiler's ability to perform register allocation.

Some use the first-class labels in the Gnu $\mathrm{C}$ compiler to goto from one function to another, e.g. the Erlang and Mercury compilers [15,26]. All code for a module is collected into a single $C$ function. Known calls within this module use ordinary gotos. A label is exported by exporting its address (a GCC extension). To branch to any unknown label, a goto is done to the address of that label (a GCC extension). Unfortunately, to make this work, severe restrictions have to placed on the generated $\mathbf{C}$ code. In particular, local $\mathrm{C}$ variables are banned: all computations must use only global variables. This has a very negative effect on the performance of the code.

The wamcc Prolog-to-C compiler [7] uses an idea similar to the previous one, but without being GCC-specific. On some RISC processors, calling a function does not in itself cause stack growth. Rather, it is the callee that extends the stack in its function prologue. The wamcc approach is to insert an assembly label at the start of every function body, and then declare a $\mathrm{C}$ function of that name. A function call to this label will therefore bypass the prologue, and hence not cause stack growth. Unfortunately, this elegant idea only works on some systems; on many others, the wamcc compiler has to insert system-dependent assembly code, making the idea inherently non-portable.

Baker [5] has proposed that tailcalls should be implemented by actual recursion. To prevent unbounded stack growth, the stack size is checked at the start of every function. When it has grown to some limit, a non-local goto (longjmp) is performed to back the stack up to an earlier point, then the aborted function is re-invoked. Also, since the $\mathrm{C}$ stack actually behaves like the allocation arena of a garbage collector, memory allocation can be performed simply by declaring local $\mathrm{C}$ variables of appropriate types. When the stack overflows, the garbage collector copies live data to a 'proper' heap. Unfortunately, the performance of this elegant scheme is extremely sensitive to the chosen stack size. Also, our measurements have indicated that it is slower than other schemes.

\footnotetext{
${ }^{4}$ The first-class labels of GCC allow this to be implemented by a jump table.
} 


\subsection{Our solution: 'dispatching switches'}

One disadvantage of the plain dispatching technique is that all calls go through the dispatcher loop, even when the target is known to be in the same module as the caller.

It is possible to compile all code in a module to a single function, and only use the dispatcher when going from one module to another, this is called 'block compilation'. Although this allows known intramodular calls to be very fast, no system we know of $[6,11]$ tries to make unknown intramodular calls efficient. Source-level 'returns' are one common source of unknown calls. In the code generated by rml2c, about $40 \%$ of all calls are unknown intramodular.

Our contribution here is a technique for efficiently testing whether an unknown call is intra- or intermodular.

First suppose that each module is compiled into a single $\mathrm{C}$ function. A label is represented as a record containing two fields: a pointer to the $\mathrm{C}$ function, and an integer tag. The $\mathrm{C}$ function takes the tag as an argument, and branches to the corresponding piece of code using a switch statement.

One strategy is to compile an unknown tailcall into a test, followed by separate code sequences for the intra- and intermodular cases. This has two problems: code size increase, and the fact that the test itself takes time. The test can be implemented either by comparing the function pointer of the target label with the current function, or by requiring different modules to use disjoint ranges of tags. The first problem can be avoided, but the test remains a bottleneck.

Our technique is the following: Let each module have a private bitmask variable. When the module is entered, its bitmask is set to all-ones, and when it is exited, the bitmask is set to zero. Also let label records have a third field, which is a pointer to its module's bitmask.

Recall that the labels are given integer tags, and that a switch is used to branch to the code when the module is entered. We reserve tag zero as a kind of trap handler. To perform an unknown tailcall, arguments are evaluated as if it was a known intramodular call, then the switch is used to branch to the bitwise 'and' of the label's tag and its bitmask variable. The code below exemplifies:

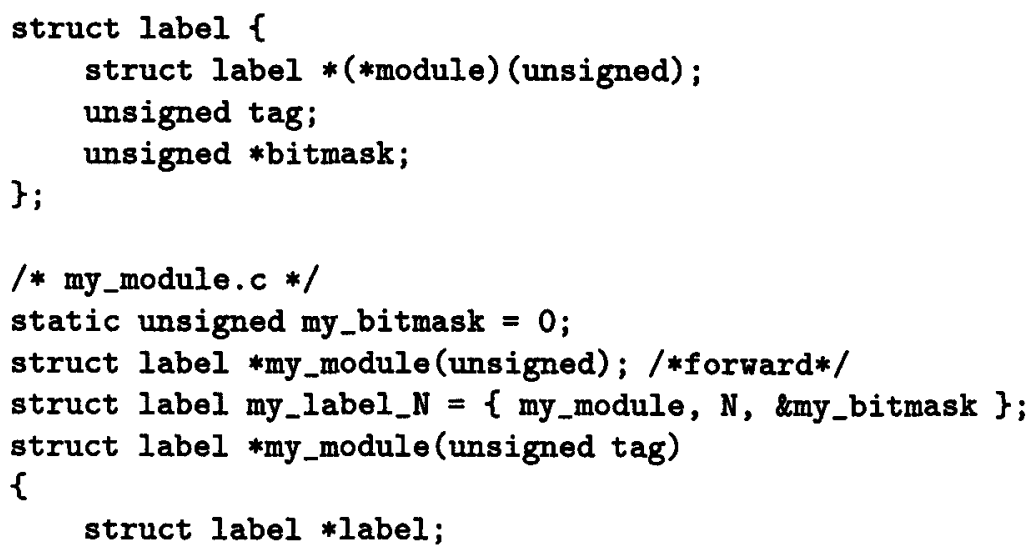




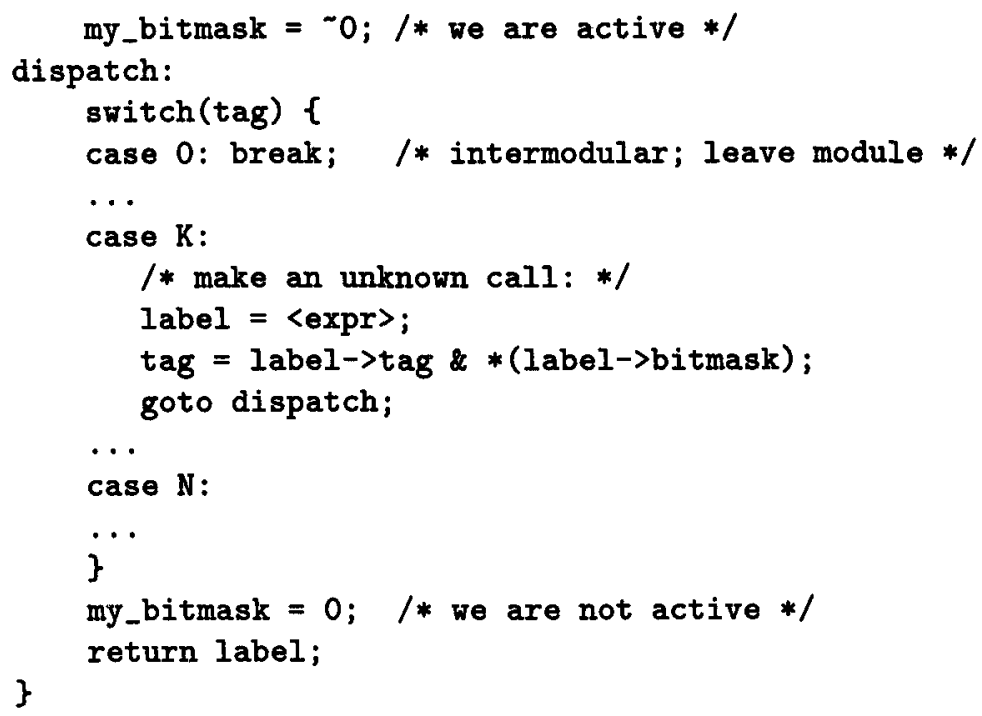

If the unknown call was intramodular, the bitmask is all-ones, and so the switch will branch to the correct label. The overhead compared to a known intramodular call is two memory loads, one 'and', and the switch ${ }^{5}$. If the call was intermodular, case zero is invoked, and the module can move parameters to global variables and return the label to the dispatcher.

This scheme has the advantage of allowing both known and unknown intramodular calls to be efficient. This is important, because of the high frequency of these cases.

\subsection{Copy-propagation}

As a consequence of tailcall emulation, global variables are used for parameter passing between modules. Even though our intermediate CPS representation is assignment-free, assignments are introduced in the translation to low-level code.

It turns out that in many label bodies, when they tailcall some other label, one or more of the outgoing (global) argument variables will have the same values as they had on entry to the label. A simple copy-propagation [1] optimization can discover when this happens, and eliminate some of the unnecessary loads and stores. (A C compiler can in general not do this optimization, since any recursive call, say to a runtime primitive, may modify any global location.)

\section{Evaluation}

To evaluate the rml2c compiler, we have performed a large number of benchmarks on several combinations of machines and $\mathrm{C}$ compilers [23].

${ }^{5}$ Usually implemented by a jump table if GCC is used. 
Our benchmark suite consisted of three RML specifications: an interpreter for a normal-order functional language applied to a prime numbers program (mf), a type checker for most of the Standard ML core language applied to a number of function definitions and a pathological expression with more than 4000 schematic type variables (miniml), and a multi-stage translator for a Pascal-like language to low-level $\mathrm{C}$ applied to a 2300 line program (petrol). These specifications represent three common application areas for natural semantics.

We have implemented four different strategies for tailcalls in rml2c. In table 1 , 'switch' is our dispatching switches strategy, 'plain' is the plain dispatching strategy, 'pushy' is Baker's recursive scheme, and 'warped' is the Erlang/Mercury use of GCC's labels. In the other dimension is listed the machines we used. Most of these are standard Unix workstations, using either their vendor's standard compiler or gcc 2.7.0/2.6.3. The 'ppc' target is a single PowerPC 601 processor in a 128-processor Parsytec GC/PP.

To do these comparisons, we had to allow the size of the 'pushy' scheme's stack buffer to vary, because of its great sensitivity to this parameter. To be fair, we also varied the size of the allocation arena used by the other schemes. In table 1 , the size was varied from $1 \mathrm{~K}$ to $128 \mathrm{~K}$ words, each benchmark was run at each step, and the sum of the execution times was computed for each tailcall scheme. Then these times were normalized with respect to the 'switch' entry.

As is evident from table 1, our dispatching switches scheme is a clear winner on all machines we have used. It is also clear that the 'pushy' scheme loses big, because of its sensitivity of the size of the stack. (Space restrictions do not allow us to report all results from this benchmark. However, our 'switch' scheme always won, and 'plain' always beat both 'pushy' and 'warped'.)

To evaluate the effectiveness of the optimizations implemented in $\mathrm{rml2c}$, we re-ran the benchmarks but with some optimizations turned off. Tables 2, 3 , and 4 list the results. In the Oall column, all optimizations are enabled. In the no-Ofol column, the FOL optimizations are disabled, and in the no-Ocps column, no optimizations were applied to the CPS level. In the last column, no-Ocode, the low-level copy propagation optimization was not performed. It is clear that optimizations applied to higher level representations, earlier in the compiler, are significantly more important for good performance than are lowerlevel optimizations.

To compare rml2c with the obvious alternatives, Typol and Prolog, we wrote Typol and Prolog versions of one of the benchmarks, viz. the mf interpreter for the normal-order functional language. In table 5 we see that Typol's performance is several orders of magnitude worse than rml2c's. Typol obviously was not implemented with efficiency in mind.

In table 6, we compare the same benchmark against two popular commercial Prolog compilers (both in native-code mode). $\mathrm{rml} 2 \mathrm{c}$ generates code that is about ten times faster than that produced by these Prolog compilers.

The petrol compiler is a natural semantics specification of a language that has been used in our department's undergraduate compiler course. We compared the performance of our rml2c generated compiler with the existing one, which 


\begin{tabular}{|l|r|r|r|r|}
\cline { 2 - 5 } \multicolumn{1}{c|}{} & switch & plain & pushy & warped \\
\hline alpha-cc & 1.00 & 1.17 & 3.40 & n/a \\
alpha-gcc & 1.00 & 1.34 & 3.00 & 1.62 \\
hp-cc & 1.00 & 1.56 & 1.95 & n/a \\
hp-gcc & 1.00 & 1.59 & 2.03 & 1.62 \\
i586-cc & 1.00 & 1.18 & 2.07 & n/a \\
mips-cc & 1.00 & 1.13 & 1.45 & n/a \\
mips-gcc & 1.00 & 1.15 & 1.30 & 1.54 \\
ppc-cc & 1.00 & 1.27 & 1.97 & n/a \\
ppc-gcc & 1.00 & 1.27 & 1.88 & 1.40 \\
sparc-cc & 1.00 & 1.17 & 3.20 & n/a \\
sparc-gcc & 1.00 & 1.20 & 1.50 & 1.41 \\
\hline \hline average & 1.00 & 1.28 & 2.16 & 1.52 \\
\hline
\end{tabular}

Table 1. Normalized time as a function of tailcall strategy

\begin{tabular}{|r|r|r|r|}
\hline Oall & no-Ofol & no-Ocps & no-Ocode \\
\hline 1.00 & 2.93 & 1.35 & 1.06 \\
\hline
\end{tabular}

Table 2. Normalized time as a function of optimization level

is written in Sun Pascal. As table 7 shows, even though the Pascal version was compiled with all optimizations enabled, it is actually $39 \%$ slower than the generated compiler.

\section{Related Work}

There are extremely few implementations of compilers for natural semantics specifications. We have already mentioned the most well-known one, the Typol compiler in the Centaur programming environment. This compiler works by translating Typol to Prolog. As our benchmarks have shown, this implementation is not efficient enough for practical use.

\begin{tabular}{|r|r|r|}
\hline Oall & no-Ofol & no-Ocps \\
\hline 1.00 & 2.25 & 2.08 \\
\hline
\end{tabular}

Table 3. Normalized \#calls as a function of optimization level 


\begin{tabular}{|r|r|r|}
\hline Oall & no-Ofol & no-Ocps \\
\hline 1.00 & 2.00 & 1.51 \\
\hline
\end{tabular}

Table 4. Normalized max stack as a function of optimization level

\begin{tabular}{|r|r|r|r|}
\hline primes & RML & TYPOL & T/R \\
\hline 3 & 0.0026 & 13 & 5000 \\
4 & 0.0037 & 72 & 19459 \\
5 & 0.0063 & 1130 & 179365 \\
\hline
\end{tabular}

Table 5. MF, RML vs. TYPOL, time in seconds

Recently, Attali and Parigot [4] have described a system that translates a sub-class of natural semantics into strongly non-circular attribute grammars. By then using the FNC-2 system's compiler for this class, they are able to improve performance by a factor of 10 compared to Typol.

A description of an early prototype of $\mathrm{rml2c}$ was published in [22]. At that time, the language had a full Prolog-like search rule, but no attempts were made to reduce non-determinism. Both the left-factoring transformations and the new implementation technique for tailcalls are later extensions.

Transformations similar to our left-factoring ones have been described before, e.g. by da Silva [8], Hannan [14], and others. As far as we know, no one has made use of them in an actual implementation before.

\section{Conclusion}

We have described a simple yet very effective compilation strategy for natural semantics. We have shown the great importance of applying high-level determinacy-improving optimizations early in the compiling process.

We have also shown that using a continuation-passing style intermediate

\begin{tabular}{|r|r|r|r|r|r|l|}
\hline primes & RML & SICStus & S/R & Quintus & Q/R & machine \\
\hline 18 & 0.45 & 5.0 & 11.1 & 4.5 & 10.0 & Sun 4/470 \\
\hline \hline 18 & 0.22 & 2.20 & 10.0 & & & Sun 10/40 \\
30 & 0.87 & 11.20 & 12.9 & & & Sun 10/40 \\
\hline
\end{tabular}

Table 6. MF, RML vs. Prolog, time in seconds 


\begin{tabular}{|r|r|r|}
\hline RML & Pascal & P/R \\
\hline 1.06 & 1.47 & 1.39 \\
\hline
\end{tabular}

Table 7. Petrol, RML vs. Pascal, time in seconds

representation can be both practical and effective in a compiler for a logicprogramming language.

A new technique, 'dispatching switches', for compiling tailcalls to efficient $\mathrm{C}$ code has been described. Our benchmarks indicate that this technique is better than several known portable and non-portable alternatives.

Our benchmarks show that rml2c generates much better code than some commercial Prolog compilers, and even better code than one commercial Pascal compiler. This clearly supports our choice of compilation strategy.

\section{References}

1. A. V. Aho, R. Sethi, and J. D. Ullman. Compilers Principles, Techniques, and Tools. Addison-Wesley, 1986.

2. Hassan AïtKaci. Warren's Abstract Machine: A Tutorial Reconstruction. The MIT Press, 1991.

3. Andrew W. Appel. Compiling with Continuations. Cambridge University Press, 1992.

4. Isabelle Attali and Didier Parigot. Integrating natural semantics and attribute grammars: the Minotaur system. Research report $\mathrm{N}^{\circ} 2339$, INRIA, September 1994.

5. Henry G. Baker. CONS should not CONS its arguments, part II: Cheney on the M.T.A. ACM SIGPLAN Notices, 30(9):17-20, September 1995.

6. Takashi Chikayama, Tetsuro Fujise, and Daigo Sekita. A portable and efficient implementation of KL1. In Hermenegildo and Penjam [16], pages 25-39.

7. Philippe Codognet and Daniel Diaz. wamcc: Compiling Prolog to C. In Leon Sterling, editor, Proceedings of the Twelfth International Conference on Logic Programming, pages 317-331, Tokyo, Japan, 1995. The MIT Press.

8. Fabio Q. B. da Silva. Towards a formal framework for evaluation of operational semantics. Technical Report ECS-LFCS-90-126, University of Edinburgh, 1990.

9. Joëlle Despeyroux. Proof of translation in natural semantics. In Proceedings of the 1st Symposium on Logic in Computer Science, LICS'86, pages 193-205. IEEE, 1986.

10. Thierry Despeyroux. Executable specification of static semantics. In Gilles Kahn, editor, Semantics of Data Types, volume 173 of $L N C S$, pages 215-233. SpringerVerlag, 1984.

11. Marc Feeley. Gambit-C version 2.2. Available by anonymous ftp from ftp.iro.umontreal.ca, May 1995.

12. Jean H. Gallier. Logic for Computer Science. John Wiley \& Sons, 1987.

13. David Gudeman, Koenraad De Bosschere, and Saumya K. Debray. jc: An efficient and portable sequential implementation of Janus. In Krzysztof Apt, editor, 
Proceedings of the Joint International Conference and Symposium on Logic Programming, pages 399-413, Washington, USA, 1992. The MIT Press.

14. John Hannan. Investigating a proof-theoretic meta-language for functional programs. Report 91/1, DIKU Copenhagen, 1991. PhD thesis.

15. Bogumil Hausman. Turbo Erlang: Approaching the speed of C. In E. Tick and G. Succi, editors, Implementations of Logic Programming Systems, pages 119-135. Kluwer Academic Publishers, 1994.

16. M. Hermenegildo and J. Penjam, editors. Proceedings of the 6th International Symposium on Programming Language Implementation and Logic Programming, PLILP'94, volume 844 of $L N C S$. Springer-Verlag, 1994.

17. I. Jacobs. The Centaur 1.2 Manual, 1992. Available from INRIA - Sophia Antipolis.

18. Gilles Kahn. Natural semantics. In F. J. Brandenburg, G. Vidal-Naquet, and M. Wirsing, editors, Proceedings of the Symposium on Theoretical Aspects of Computer Science, STACS'87, volume 247 of LNCS, pages 22-39. Springer-Verlag, 1987.

19. David Kranz, Richard Kelsey, Jonathan A. Rees, Paul Hudak, James Philbin, and Norman I. Adams. Orbit: an optimizing compiler for Scheme. In Proceedings of the ACM SIGPLAN '86 Symposium on Compiler Construction, pages 219-233. ACM Press, 1986.

20. Robin Milner, Mads Tofte, and Robert Harper. The Definition of Standard ML. The MIT Press, 1990.

21. Mikael Pettersson. A term pattern-match compiler inspired by finite automata theory. In U. Kastens and P. Pfahler, editors, Compiler Construction, 4th International Conference, CC'92, volume 641 of $L N C S$, pages 258-270. Springer-Verlag, October 1992.

22. Mikael Pettersson. RML - a new language and implementation for natural semantics. In Hermenegildo and Penjam [16], pages 117-131.

23. Mikael Pettersson. Compiling Natural Semantics. PhD thesis, Department of Computer and Information Science, Linköping University, December 1995.

24. Simon L. Peyton Jones. Implementing lazy functional languages on stock hardware: the spineless tagless G-machine. Journal of Functional Programming, 2(2):127-202, April 1992.

25. Gordon D. Plotkin. A structural approach to operational semantics. Report DAIMI FN-19, Computer Science Department, Aarhus University, Denmark, September 1981.

26. Zoltan Somogyi, Fergus James Henderson, and Thomas Charles Conway. The implementation of Mercury, an efficient purely declarative logic programming language. In Koen De Bosschere, Bart Demoen, and Paul Tarau, editors, ILPS'94 Post-Conference Workshop on Implementation Techniques for Logic Programming Language, pages 31-58, 1994.

27. Guy L. Steele Jr. Rabbit: a compiler for Scheme (a study in compiler optimization). MIT AI Memo 474, Massachusetts Institute of Technology, May 1978. Master's Thesis.

28. David R. Tarditi, Peter Lee, and Anurag Acharya. No assembly required: Compiling Standard ML to C. ACM LOPLAS, 1(2):161-177, June 1992. 